Check for updates

Cite this: RSC Adv., 2022, 12, 7540

\title{
Cinnamaldehyde-cucurbituril complex: investigation of loading efficiency and its role in enhancing cinnamaldehyde in vitro anti-tumor activity
}

\author{
Bayan Al Tbakhi, ${ }^{a}$ Hamdi Nsairat, (D) ${ }^{b}$ Walhan Alshaer, (D) ${ }^{c}$ Abdulfattah Al-Kadash, (DD d \\ Wissam Helal, (D) a Lubna Alrawashdeh, ${ }^{e}$ Anthony Day, (D) ${ }^{f}$ Khaleel I. Assaf, (D) ${ }^{g}$ \\ Rola Hassouneh, ${ }^{a}$ Fadwa Odeh (D) *a and Abeer Al Bawab*ah
}

\begin{abstract}
This study aimed to clarify the physico-chemical properties of cucurbit[7]uril (CB[7]) and cinnamaldehyde (Cinn) inclusion complexes (CB[7]-Cinn) and their resulting antitumor activity. CB[7]-Cinn inclusion complexes were prepared by a simple experimental approach and fully characterized for their stoichiometry, formation constant, particle size and morphology. Quantum chemical calculations were performed to elucidate the stable molecular structures of the inclusion complexes and their precursors and to investigate the probable stoichiometry and direction of interaction using three different DFT functionals at the $6-31 G(d, p)$ basis set. The UV-vis spectrophotometric titrations as well as the Job plot, based on ${ }^{1} \mathrm{H}$ NMR spectroscopy, suggested $1: 1$ and $1: 2$ stoichiometries of CB[7] : Cinn. The formation constants of the complexes were calculated using Benesi-Hildebrand equations and non-linear fittings. Moreover, the theoretical calculations confirmed the potential formation of $1: 1$ and $1: 2$ stoichiometries and clarify the orientation of binding from the Cinn phenyl moiety. The nanoparticles' TEM images showed a crystal-like spherical shape, smooth surface, with a small tendency to agglomerate. CB[7]Cinn inclusion complexes were analyzed for their antitumor activity against MDA-MB-231 breast cancer and U-87 glioblastoma cell lines. The $I C_{50}$ values were calculated after 72 hours of incubation with different concentrations of CB[7]-Cinn inclusion complexes and compared to free Cinn and free CB[7]. The $I_{50}$ values for free Cinn and CB[7]-Cinn inclusion complexes were $240.17 \pm 32.46 \mu \mathrm{M}$ and 260.47 $\pm 20.83 \mu \mathrm{M}$ against $\mathrm{U}-87$ cells and $85.93 \pm 3.35 \mu \mathrm{M}$ and $176.3 \pm 7.79 \mu \mathrm{M}$ against MDA-MB-231 cells, respectively, despite the enhanced aqueous solubility. No significant cytotoxicity was noticed for the free $\mathrm{CB}[7]$.
\end{abstract}

Received 4th January 2022

Accepted 1st March 2022

DOI: $10.1039 / \mathrm{d} 2 \mathrm{ra00044j}$

rsc.li/rsc-advances

\section{Introduction}

Most cytotoxic anticancer drugs are organic compounds with low aqueous solubility, are unstable, and have poor bioavailability. ${ }^{1}$ To overcome these drawbacks, nanomedicine offers solutions to enhance water solubility and minimize drug

a Department of Chemistry, School of Science, The University of Jordan, Amman 11942, Jordan. E-mail: f.odeh@ju.edu.jo; Tel: +962-979250409

${ }^{b}$ Pharmacological and Diagnostic Research Centre, Faculty of Pharmacy, Al-Ahliyya Amman University, Amman 19328, Jordan

'Cell Therapy Center, The University of Jordan, Amman 11942, Jordan

${ }^{d}$ School of Medicine, The University of Jordan, Amman 11942, Jordan

${ }^{e}$ Department of Chemistry, Faculty of Science, The Hashemite University, P. O. Box 330127, Zarqa 13133, Jordan

${ }^{f}$ School of Science, The University of New South Wales, Canberra, ACT 2600, Australia ${ }^{g}$ Department of Chemistry, Al-Balqa Applied University, Al-Salt 19117, Jordan

${ }^{h}$ Hamdi Mango Center for Scientific Research, The University of Jordan, Amman 11942, Jordan. E-mail: drabeer@ju.edu.jo; Tel: +962-796661601 toxicity. ${ }^{2}$ Cinnamaldehyde (Cinn, (2E)-3-phenylprop-2-enal, Fig. 1a), the main active component of cinnamon oil, is a viscous liquid effective as an antibacterial, anti-inflammation, antivirus, and anticancer agent. ${ }^{3-8}$ However, Cinn has low aqueous solubility and is easily oxidized by air, ${ }^{9}$ thus limiting its pharmacological usage and bioavailability. To improve the pharmaceutical properties and enhance the aqueous solubility and stability and/or reduce drugs' toxicity of lipophilic potential
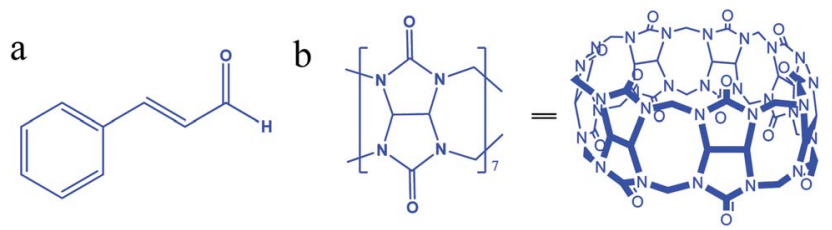

Fig. 1 Chemical structure of (a) cinnamaldehyde and (b) cucurbit[7] uril. 
drugs, synthetic or naturally occurring complexing hosts, such as cyclodextrins (CDs), crown ethers, and calixarenes, have been extensively investigated and used as drug carriers. ${ }^{10,11}$ Most drug delivery systems depended on the ability of certain hydrophilic macrocyclic compounds to act as a molecular carrier for lipophilic drugs.

Cinn complexes with cyclodextrins and their derivatives were synthesized and investigated. ${ }^{12-17}$ However, other macrocyclic compound, such as cucurbit[7]uril (Fig. 1b), may act as drug carriers.

Cucurbit $[n]$ urils (CB $[n])$, a new family of synthetic macrocyclic hosting agents composed of glycoluril (Fig. 1b) repeated units to form a molecular container. ${ }^{18}$ The $\mathrm{CB}[n]$ family members have common characteristics, such as a hydrophobic cavity and two identical opening hydrophilic entrances. In addition, the varying cavity and portal sizes lead to the formation of inclusion complexes with different molecules through a combination of ion-dipole, hydrogen bonding, and hydrophobic interactions. ${ }^{19}$ Toxicity studies proved that $\mathrm{CB}[n] \mathrm{s}$ are biocompatible with low toxicity in the intravenous and oral administration routes. ${ }^{20}$ The host-guest features of $\mathrm{CB}[n]$ have a wide range of applications in nanomachines, self-assembly, drug delivery, the removal of wastes, and in chromatography. ${ }^{19}$ Moreover, the reversibility of the host-guest complexation process allows full or partial binding of the biological or medicinal activity of the complexed pharmaceutical agents. ${ }^{8}$

Among $\mathrm{CB}[n] \mathrm{s}, \mathrm{CB}[6]$ and $\mathrm{CB}[8]$ are almost insoluble in water, whereas $\mathrm{CB}[7]$ has a moderate solubility $(2 \times$ $10^{-2} \mathrm{~mol} \mathrm{~L}^{-1}$ ), which is similar to the commonly used complexing host, $\beta$-cyclodextrin (with a solubility of $1.6 \times$ $10^{-2} \mathrm{~mol} \mathrm{~L}^{-1}$ in water). Moreover, the suitable size of cavity $(7.3$ $\AA$ diameter) ${ }^{21}$ symmetrical portals (5.4 A diameter) and the $9.1 \AA$ height of $\mathrm{CB}[7]$ make it suitable for binding certain drug molecules, such as Cinn which has a length of $8.3 \AA$ and a height of $4.9 \AA^{10}$

Cinn is a planar molecule consisting of one aromatic ring (phenyl) connected with an aliphatic arm of three carbon atoms ending with an aldehyde. The encapsulation of Cinn into host molecules (such as $\beta$-cyclodextrin) is usually through the nonpolar part of the molecules (the phenyl and aryl), ${ }^{14}$ which would enhance its water solubility, protect it from oxidation, increase its stability and may-as a potential drug-improve its activity.

In this study, we prepared and characterized $\mathrm{CB}[7]-\mathrm{Cinn}$ inclusion complex using optical and spectroscopic techniques in addition to electron microscopic imaging. Moreover, a theoretical evaluation of the $\mathrm{CB}[7]-$ Cinn inclusion complex stoichiometry and energy measurements was performed. The antitumor activity of $\mathrm{CB}[7]-\mathrm{Cinn}$ inclusion complex against MDA231 and U87 cancer cell lines was also investigated.

\section{Materials and methods}

\subsection{Chemicals}

trans-Cinn (99\%) was purchased from Sigma-Aldrich (USA). Cucurbit[7]uril ( $\mathrm{CB}[7])$ was prepared according to literature. ${ }^{22}$ $\mathrm{D}_{2} \mathrm{O}$ (99 atom\% D) was purchased from Sigma-Aldrich (USA), and deionized water was prepared using Osmo Aries device (USA). Uranyl acetate reagent was obtained from Electron Microscopy Science, USA. All reagents and chemicals were used without further treatment.

\subsection{The formation of $\mathrm{CB}[7]$-Cinn inclusion complexes}

2.2.1. $\quad{ }^{1}$ H-NMR study. All NMR spectra were performed in deuterium oxide $\left(\mathrm{D}_{2} \mathrm{O}, 99.0\right.$ atom\% D, Sigma-Aldrich, USA $)$ using Bruker Biospin AG Magnet system $500 \mathrm{MHz} / 54 \mathrm{~mm}$ instrument (Bruker BioSpin, Switzerland) with PA BBO 500S1 BBF-H-D-05 Z SP probe and the temperature was controlled using a variable temperature unit (VTU) and held constant at $298 \mathrm{~K}$. Data processing and analysis was performed using topspin 4.0.2 (Bruker Biospin GmbH, Switzerland).

\section{3. $\mathrm{CB}[7]-\mathrm{Cinn}$ complexation stoichiometry and formation constants}

2.3.1. Spectrophotometric studies. Spectrophotometric studies were carried out to determine the absorption spectra, stoichiometry and formation constant of the $\mathrm{CB}[7]-\mathrm{Cinn}$ inclusion complexes. Absorption spectra were recorded in the range 200-400 $\mathrm{nm}$ where the $\lambda_{\max }$ was determined at $272 \mathrm{~nm}$ for Cinn aqueous solution. Since Cinn has low water solubility, the spectral data were performed using a fixed concentration of supersaturated Cinn solution with increasing amounts of CB [7]. ${ }^{23}$ Uncomplexed Cinn solution (Cinn aqueous solution only) was used as the control reference. Cinn with $\mathrm{CB}$ [7] UV-vis spectrophotometry titrations for Benesi-Hildebrand experiments were performed by maintaining the concentration of Cinn constant $(0.06 \mathrm{mM})$ and the $\mathrm{CB}[7]$ concentration was varied from $0.03-0.15 \mathrm{mM}$. All spectrophotometric measurements were performed using Nanodrop 2000 UV-vis spectrophotometer (Wilmington, DE, USA).

The stoichiometry and the formation constants of $\mathrm{CB}[7]-$ Cinn inclusion complex were determined using the BenesiHildebrand equations ${ }^{24}$ based on the data from UV-vis spectrophotometry. Eqn (1) was used for $1: 1$ complexation and eqn (2) for $1: 2$ complexation. ${ }^{25-28}$

$$
\begin{aligned}
& \frac{1}{Y-Y^{\mathrm{o}}}=\frac{1}{Y^{\prime}-Y^{\mathrm{o}}}+\frac{1}{K\left(Y^{\prime}-Y^{\mathrm{o}}\right)[Z]} \\
& \frac{1}{Y-Y^{\mathrm{o}}}=\frac{1}{Y^{\prime}-Y^{\mathrm{o}}}+\frac{1}{K\left(Y^{\prime}-Y^{\mathrm{o}}\right)[Z]^{2}}
\end{aligned}
$$

According to the design of Benesi-Hildebrand experiment, $Z$ is the increasing concentration of either the host or the guest, $Y^{\circ}$ is absorbance $\left(A^{\circ}\right)$ of Cinn. $Y$ is absorbance $(A)$ with a particular concentration of either the host or the guest, $Y^{\prime}$ is absorbance $\left(A^{\prime}\right)$ at the maximum concentration of either the host or the guest. The formation constant $(K)$ is calculated from the BenesiHildebrand equation, ${ }^{26}$ after plotting $\left(1 / Y-Y^{\circ}\right)$ against $(1 /[Z])$ or $\left(1 /[Z]^{2}\right)$ :

$$
\text { Formation constant }(K)=\frac{1}{\text { slope }\left(Y^{\prime}-Y^{\circ}\right)}
$$


The continuous variation method (Job's method) was also used to determine the complexation stoichiometry ${ }^{24,29}$ using ${ }^{1} \mathrm{H}$ NMR spectroscopy. The experiments were performed using solutions of equimolar concentrations of the Cinn and $\mathrm{CB}[7]$. The samples were prepared by mixing different volumes of these two solutions so that the total concentration remains constant and the molar fraction of Cinn or $\mathrm{CB}[7], \chi$ varies in the range $0-1$. The chemical shift differences in the presence of $\mathrm{CB}$ [7] in respect to the value for the free Cinn, was determined. The variation of the chemical shifts $(\Delta \delta)$ multiplied by Cinn or $\mathrm{CB}[7]$ molar ratio, is plotted versus Cinn or $\mathrm{CB}[7]$ mole fraction $(\chi)$. The value of $\chi$ for which the plot presents the maximum deviation gives the stoichiometry of the inclusion complex $(\chi=0.5$ for $1: 1$ or $2: 2$ ratio complexes; $\chi=0.33$ for $1: 2$ ratio complexes). ${ }^{30}$

\subsection{Dynamic light scattering (DLS)}

Nanoparticles size of the $\mathrm{CB}[7]$ : Cinn inclusion complex of $1: 1$ ratio $(1 \mathrm{mM})$ was determined by dynamic light scattering (DLS) (NICOMP, USA). All samples were diluted at room temperature $\left(25{ }^{\circ} \mathrm{C}\right)$ with deionized water in order to yield an appropriate counting rate. ${ }^{31}$ The stability of the nanoparticles was determined by measuring the sizes at different time points.

\subsection{Electron microscopy (EM)}

An aqueous solution of the optimum ratio (1:1) of $\mathrm{CB}[7]-\mathrm{Cinn}$ inclusion complexes was examined for the structure and morphology using a MORGAGNI 268-FEI Transmission Electron Microscope (TEM), (Netherlands).

TEM analysis was accomplished by placing $7 \mu \mathrm{L}$ of the diluted aqueous suspensions $(1 \% \mathrm{v} / \mathrm{v})$ on 300-mesh formvar copper grids (SPI supplies, USA) and left to dry overnight. The loaded grids were then stained using $2 \%$ uranyl acetate dye solution. Excess liquid on the grid was removed with filter paper and the grid was allowed to dry before being observed under Versa 3D (FEI, Netherlands) operated at $30 \mathrm{kV}$.

\subsection{Computational methodology}

All quantum mechanical calculations were performed using the Gaussian 09 program package. ${ }^{32}$ The molecular structures and geometries of free $\mathrm{CB}[7]$ and Cinn were optimized at the DFT level of theory using the Becke, 3-parameter, Lee-Yang-Parr (B3LYP) functional with 6-31G(d,p) basis set.

The solvent effects were taken into account by using the conductor-like polarizable continuum model (CPCM) method with water as a solvent. Frequency calculations were performed on the optimized geometries at the same level of theory. All computed vibrational transitions have no imaginary frequency implying that each optimized geometry is located at the global minimum point on the potential energy surface. In the second step, an optimization procedure was performed in order to elucidate the stability of the $1: 1$ complex as a function of the direction of the aldehyde arm toward the macrocycle cavity at constant distances between both compounds. Subsequently, the $1: 1$ complex potential energy surface was modeled by freezing all geometry parameters, while scanning the single point energy of the complex as a function of the distance between Cinn and the center of the $\mathrm{CB}$ [7] macrocycle cavity, with intervals of $0.250 \AA$. For this step, three different hybrid functionals were used, namely: B3LYP, PBE0, and M06-2X. Finally, a second Cinn molecule was incorporated into the $1: 1$ complex and a new potential energy curve was computed by freezing all geometry parameters, while scanning the single point energy of the complex as a function of the distance between the second Cinn and the center of the $\mathrm{CB}[7]$ cavity, with intervals of $0.250(\AA)$. All DFT calculations were applied to both sides of Cinn compound, i.e. sides of inclusion in $\mathrm{CB}[7]$ host (phenyl side and aldehyde side).

\subsection{In vitro cytotoxicity assay}

Cytotoxic activity tests were performed against human breast adenocarcinoma (MDA-MB-231, ATCC number: HTB26) and human primary glioblastoma (U87, ATCC number: HTB-14) for $\mathrm{CB}[7]-\mathrm{Cinn}$ inclusion complexes and their free precursors, Cinn and $\mathrm{CB}[7]$. Cells were cultured in Dulbecco's Modified Eagle's Medium (DMEM) growth medium (Euroclone®, Italy) supplemented with $10 \%(\mathrm{v} / \mathrm{v})$ fetal bovine serum (FBS), 1\% (v/v) $200 \mathrm{mM}$ L-glutamine, (100 IU mL $\mathrm{mL}^{-1}-100 \mu \mathrm{g} \mathrm{mL}^{-1}$ ) penicillinstreptomycin. Cells were maintained in a humidified $5 \% \mathrm{CO}_{2}$ incubator at $37{ }^{\circ} \mathrm{C}$ and used at a confluence of $75-95 \%$.

A total of $5 \times 10^{3}$ cells per well were seeded in 96-well plates (TPP, Switzerland) at $37{ }^{\circ} \mathrm{C}$ and $5 \% \mathrm{CO}_{2} \cdot{ }^{33}$ After 24 hours, cells were treated with different concentrations of $\mathrm{CB}[7]-\mathrm{Cinn}$ inclusion complexes and their free precursors, Cinn and $\mathrm{CB}[7]$ up to $900 \mu \mathrm{M}$. The cell growth was analyzed using tetrazolium dye (MTT; 3[4,5-dimethylthiazol-2-yl]-2,5-diphenyltetrazolium bromide) assay ${ }^{33,34}$ after 72 hours. Untreated cells were considered as a negative control and were used as blank to calibrate the spectrophotometer, then the absorbance was read at $570 \mathrm{~nm}$ using a using Glomax plate reader (Promega, USA). IC $_{50}$ values were calculated using GraphPad Prism 6 (GraphPad Software Inc., USA). All experiments were performed in triplicate.

\section{Results and discussion}

\subsection{Formation of $\mathrm{CB}[7]-\mathrm{Cinn}$ inclusion complex}

3.1.1. ${ }^{1}$ H-NMR study. NMR is a powerful technique in studying inclusion complex formation since it is capable of monitoring the variations in microenvironments due to changes in intermolecular interaction for each proton. ${ }^{28}{ }^{1} \mathrm{H}$ NMR spectrum of $\mathrm{CB}$ [7] has two doublets $\mathrm{CH}_{2}$ (a) and $\mathrm{H}_{2}$ (b) for the $\mathrm{CH}_{2}$ protons at 4.09 and $5.66 \mathrm{ppm}$ and a singlet for $\mathrm{CHc}$ protons at $5.39 \mathrm{ppm}$ (Fig. 2a). The ${ }^{1} \mathrm{H}-\mathrm{NMR}$ spectrum for Cinn consists of two doublet protons, $\mathrm{H} 1$ and $\mathrm{H} 3$ at 9.46 and $7.68 \mathrm{ppm}$, three multiplets protons, $\mathrm{H} 4, \mathrm{H} 5$ and $\mathrm{H} 6$ at 7.62, 7.41 and $7.39 \mathrm{ppm}$, respectively, and a doublet of doublets $\mathrm{H} 2$ protons at 6.57 ppm (Fig. 2b).

The formation of the $\mathrm{CB}[7]-\mathrm{Cinn}$ inclusion complex was clearly evidenced by the chemical shift changes experienced by Cinn protons upon the addition of increasing amounts of $\mathrm{CB}$ [7] as shown in Fig. 3. The upfield shift of Cinn protons in the 

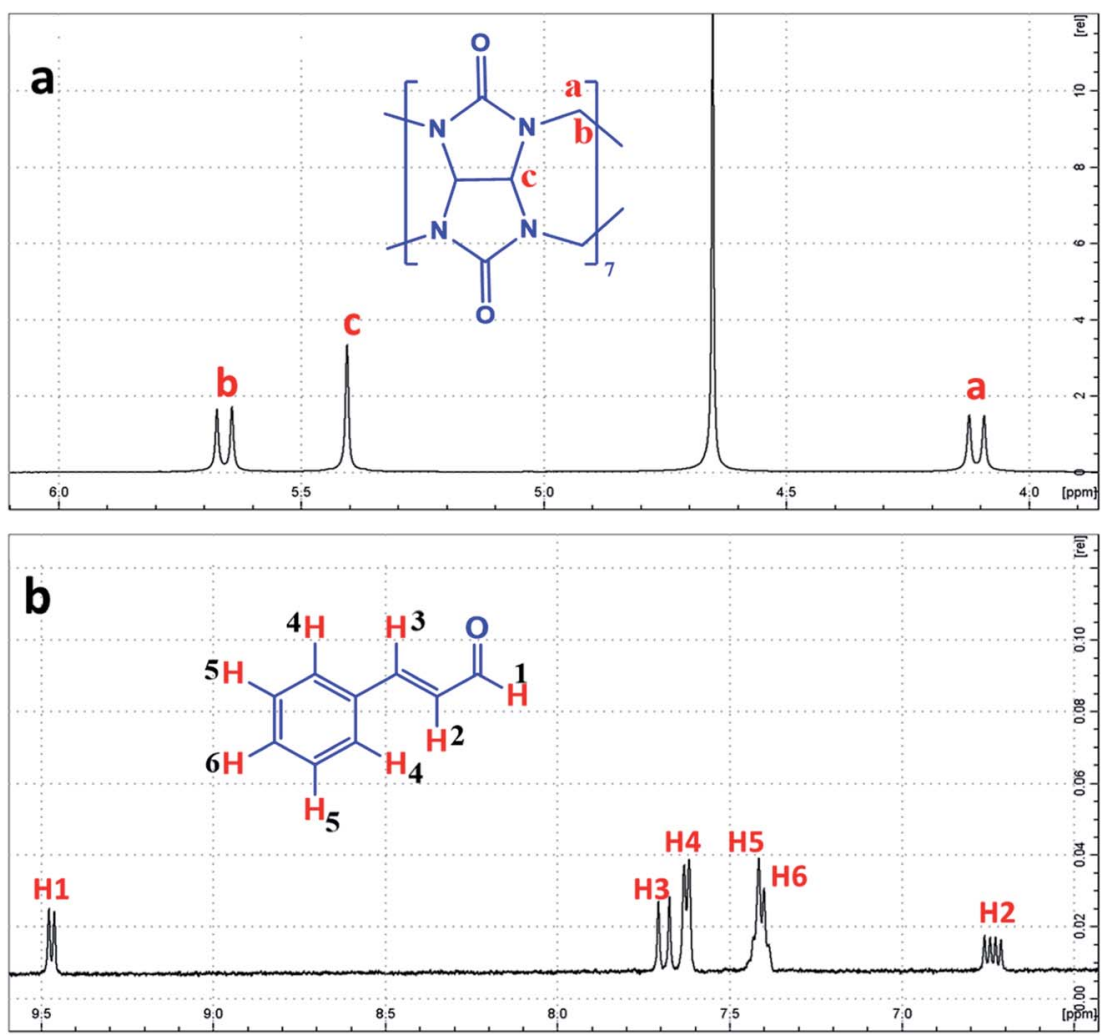

Fig. $2{ }^{1} \mathrm{H}$-NMR spectrum of (a) $\mathrm{CB}[7]$ and (b) $\mathrm{Cinn}$, in $\mathrm{D}_{2} \mathrm{O}$ at room temperature, with the protons assigned.

inclusion complex compared to the free Cinn is an indication of a more shielded and restricted microenvironment of the Cinn molecules due to complexation. ${ }^{35}$ It is clear that the phenyl protons experienced a dramatic change both in shape and chemical shift (Fig. 3) indicating a full inclusion of Cinn in CB [7] from the benzene ring as expected. Both $\mathrm{H} 2$ and $\mathrm{H} 3$ protons underwent a considerable shift, even though it was less than the shift of the phenyl protons, whereas $\mathrm{H} 1$ (the aldehyde proton) witnessed no shift at all.

From the ${ }^{1} \mathrm{H}$-NMR spectra, it is obvious that the guest (Cinn) enters the $\mathrm{CB}[7]$ cavity from the phenyl side. These results are in good agreement with previous studies dealing with aromatic compound having the methylene side chain as reported by Sindelar and coworkers. ${ }^{36}$ They confirmed, using ${ }^{1} \mathrm{H}-\mathrm{NMR}$ titration, that all anthraquinone unit protons are located inside the $\mathrm{CB}[7]$ cavity while the methylene protons positioned outside $\mathrm{CB}[7] .^{36}$

\section{2. $\mathrm{CB}[7]-\mathrm{Cinn}$ complexation stoichiometry and formation constants}

3.2.1. Spectrophotometric studies. Spectrophotometry is one of the methods usually used to confirm inclusion complex

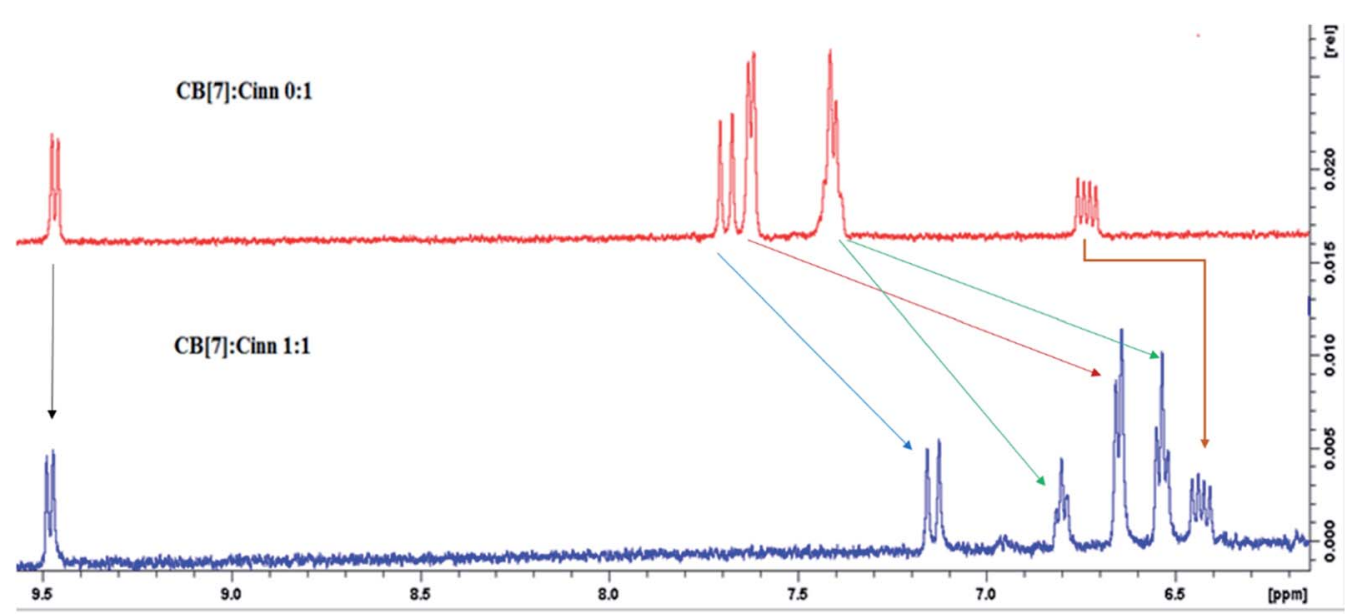

Fig. $3{ }^{1} \mathrm{H}$ NMR spectra of $1: 1 \mathrm{CB}[7]$-Cinn inclusion complex in $\mathrm{D}_{2} \mathrm{O}$ compared to the free Cinn in $\mathrm{D}_{2} \mathrm{O}$. 
a

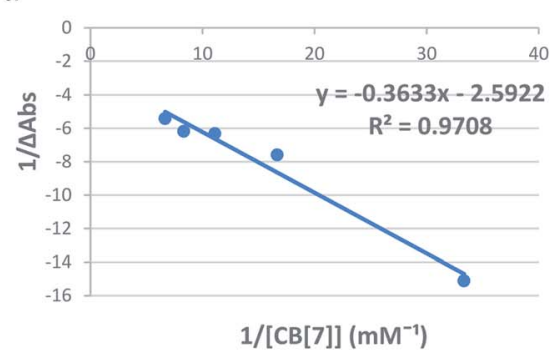

b

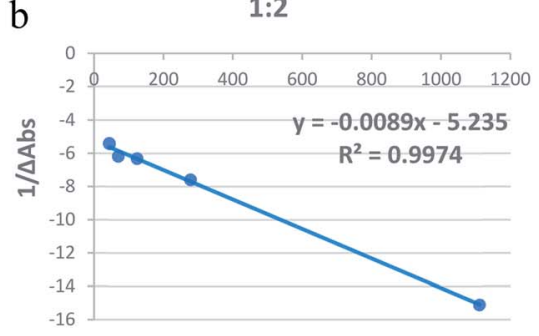

$1 /[\mathrm{CB}[7]]^{2}\left(\mathrm{mM}^{-2}\right)$

Fig. 4 Benesi-Hildebrand plots of (a) $1 / \Delta A$ versus $1 /\left[C B[7]\right.$ and (b) $1 / \Delta A$ versus $1 /[C B[7]]^{2}$ for CB[7]-Cinn inclusion complex using UV-vis spectrophotometric titration.
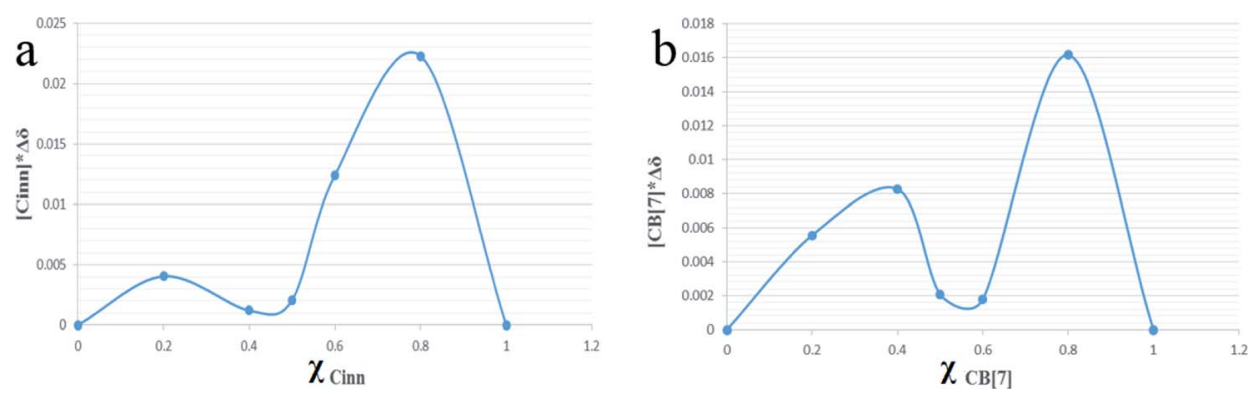

Fig. 5 Job's plot of CB[7]-Cinn inclusion complex with respect to (a) Cinn and (b) CB[7], where $\chi$ is the mole fraction.

formation. The stoichiometry was determined using the Benesi-Hildebrand equations (eqn (1) and (2)). A linear dependence of the type $1 /\left(A-A^{\circ}\right)$ vs. $1 /[\mathrm{CB}[7]]^{n}$, with $n=1$ or 2 , indicates the presence of complexes of $1: 1$ or $1: 2$ stoichiometry of $\mathrm{CB}$ [7]: Cinn, respectively. ${ }^{25}$ Fig. $4 \mathrm{a}$ and b represent the BenesiHildebrand plots for $\mathrm{CB}[7]$-Cinn inclusion complex using UVvis spectrophotometric titration for both stoichiometries. A good linear correlation was obtained for $n=1$ (Fig. 4a), indicating a $1: 1$ stoichiometry with a formation constant $\left(K_{\mathrm{f}}\right)$ of $15.13 \pm 1.99 \times 10^{3} \mathrm{M}^{-1}$. However, another good linear correlation with the possibility of a $1: 2$ stoichiometry appears from Fig. $4 \mathrm{~b}$, with a $K_{\mathrm{f}}$ of $620.05 \pm 75.45 \times 10^{3} \mathrm{M}^{-2}$. The formation of inclusion complexes with $1: 2$ (host: guest) stoichiometry is also possible. The presence of these two stoichiometric possibilities may be attributed to solvent effects, but either ratios will not affect the spectrophotometric investigations of the inclusion complex. ${ }^{37}$

The Benesi-Hildebrand plots from UV-vis spectrophotometric spectroscopic data indicate the two possibilities of the formation of complexes with different stoichiometries ( $1: 1$ and $1: 2$ and/or $2: 1$ of the CB[7]-Cinn inclusion complex). Furthermore, our results are in good consistency with previous studies. For example, Koner et al. reported a binding constant

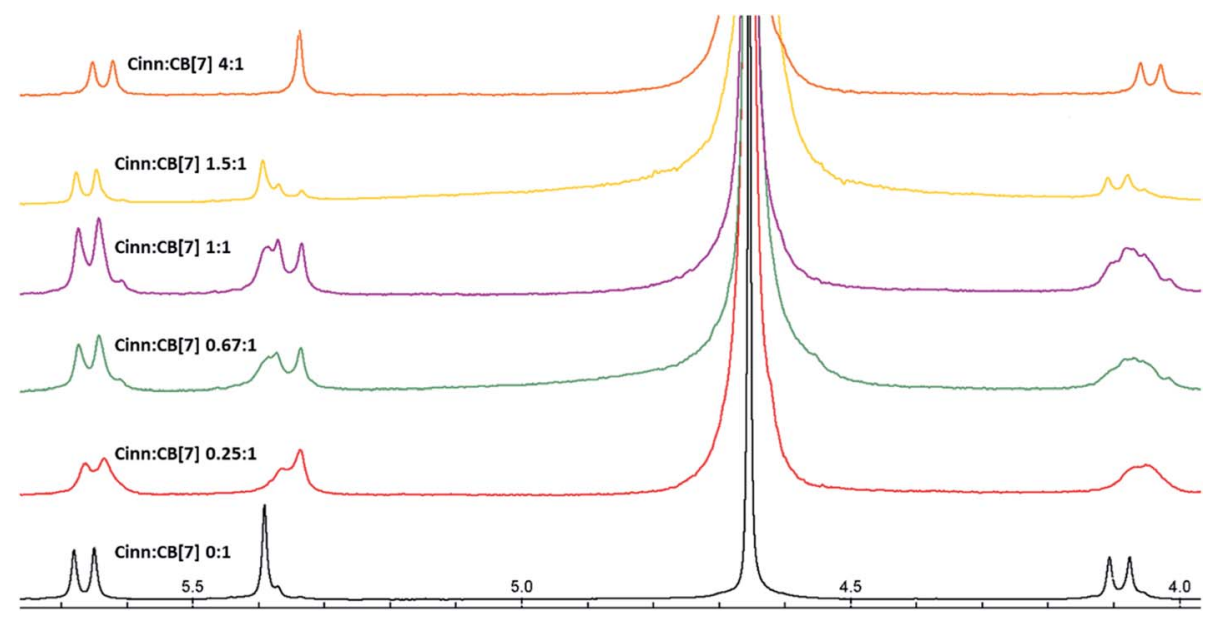

Fig. $6{ }^{1} \mathrm{H}$ NMR stacked spectra in $\mathrm{D}_{2} \mathrm{O}$ solvent of $\mathrm{Cinn}$ with $\mathrm{CB}[7]$ at different mole fractions according to Job's plot method. 
values for the neutral form of albendazole into $\mathrm{CB}$ [7] to be $6.5 \times$ $10^{4} \mathrm{M}^{-1}$ in the UV titrations with $\mathrm{CB}[7] .^{38}$

Continuous variation method (Job's method) using ${ }^{1} \mathrm{H}-\mathrm{NMR}$ titration data was also employed to determine the complexation stoichiometry. Job's plots are shown in Fig. 5a and b with respect to Cinn and $\mathrm{CB}[7]$, respectively, where the mole fraction of either the host or guest $(\chi)$ was varied from 0.0 to 1.0 and plotted against $\chi$ multiplied by the chemical shift difference of $\mathrm{H} 3$ proton of $\mathrm{CB}[7]$ (Fig. 6). As shown in Job's plot, two maximum peaks were obtained around $\chi=0.5$. These two parabola sections may point to the probability of a $1: 1$ and $1: 2$ inclusion complex between Cinn and $\mathrm{CB}[7]$ as evidenced in Benesi-Hildebrand plots. ${ }^{39,40}$ This may be attributed to the symmetrical portal sides of $\mathrm{CB}[7]$ and the small size of Cinn that led to fast equilibrium of the physical interaction between the complex precursors. The same observation was reported by Romano et al. with Cinn- $\alpha$-cyclodextrin inclusion complex. ${ }^{41}$

The possibility of the formation of different inclusion complexes is clearly suggested by ${ }^{1} \mathrm{H}$ NMR data. Fig. 6 shows the ${ }^{1} \mathrm{H}$ NMR spectrum of $\mathrm{CB}[7]$ in the presence of different concentrations of Cinn. As the Cinn concentration increases, the ' $a$ ' protons of $\mathrm{CB}$ [7] start to broaden with an upfield shift suggesting the presence of different species including empty $\mathrm{CB}$ [7], $1: 1$ and $2: 1 \mathrm{Cinn}: \mathrm{CB}[7]$ complexes. At a ratio of Cinn : CB [7] of $4: 1$, a shifted doublet exists suggesting a predominant $2: 1$ complex. The same trend is observed with 'c' protons of CB [7], a singlet at $5.39 \mathrm{ppm}$ (no Cinn present). Upon the addition of Cinn, the signal starts to split in the Cinn : $\mathrm{CB}$ [7] ratio from $0.25: 1$ to $1.5: 1$, suggesting the presence of different species. However, when the concentration of Cinn is increased to reach $4: 1$ ratio, a singlet at $5.34 \mathrm{ppm}$, suggesting a predominant $2: 1$ complex. These results suggest that an equilibrium is present between free $\mathrm{CB}[7]$, a $1: 1$ complex (I) and $2: 1$ complex with

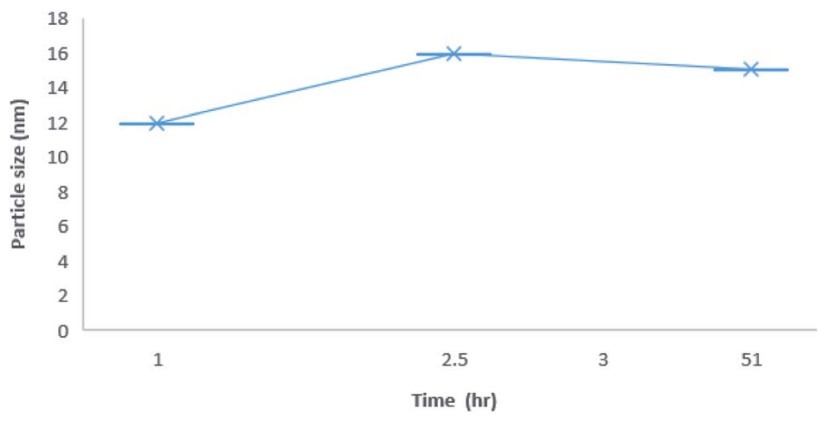

Fig. 8 Average size distribution by intensity with different time point for $1: 1$ inclusion complex nanoparticles of CB[7]-Cinn.

two possibilities (II and III) as shown in Fig. 7. At a large excess of Cinn, a $2: 1$ complex is dominant.

\subsection{Nanoparticle size measurements}

The dynamic light scattering (DLS) of $\mathrm{CB}$ [7]-Cinn inclusion complex with 1:1 stoichiometry was determined at different time intervals to assess the stability of the inclusion complexes (Fig. 8). The results showed that $\mathrm{CB}$ [7]-Cinn nanoparticles' size range from 11-30 $\mathrm{nm}$ with good stability and maintained their size stability up to 50 hours.

\subsection{Electron microscopy (EM)}

Transmission electron microscopy (TEM) image showed a highly dispersed dry $\mathrm{CB}[7]$-Cinn nanoparticles in the range of 10-20 nm size (Fig. 9). This size range is considerably smaller than hydrodynamic sizes obtained by the dynamic light scattering (DLS) experiments.

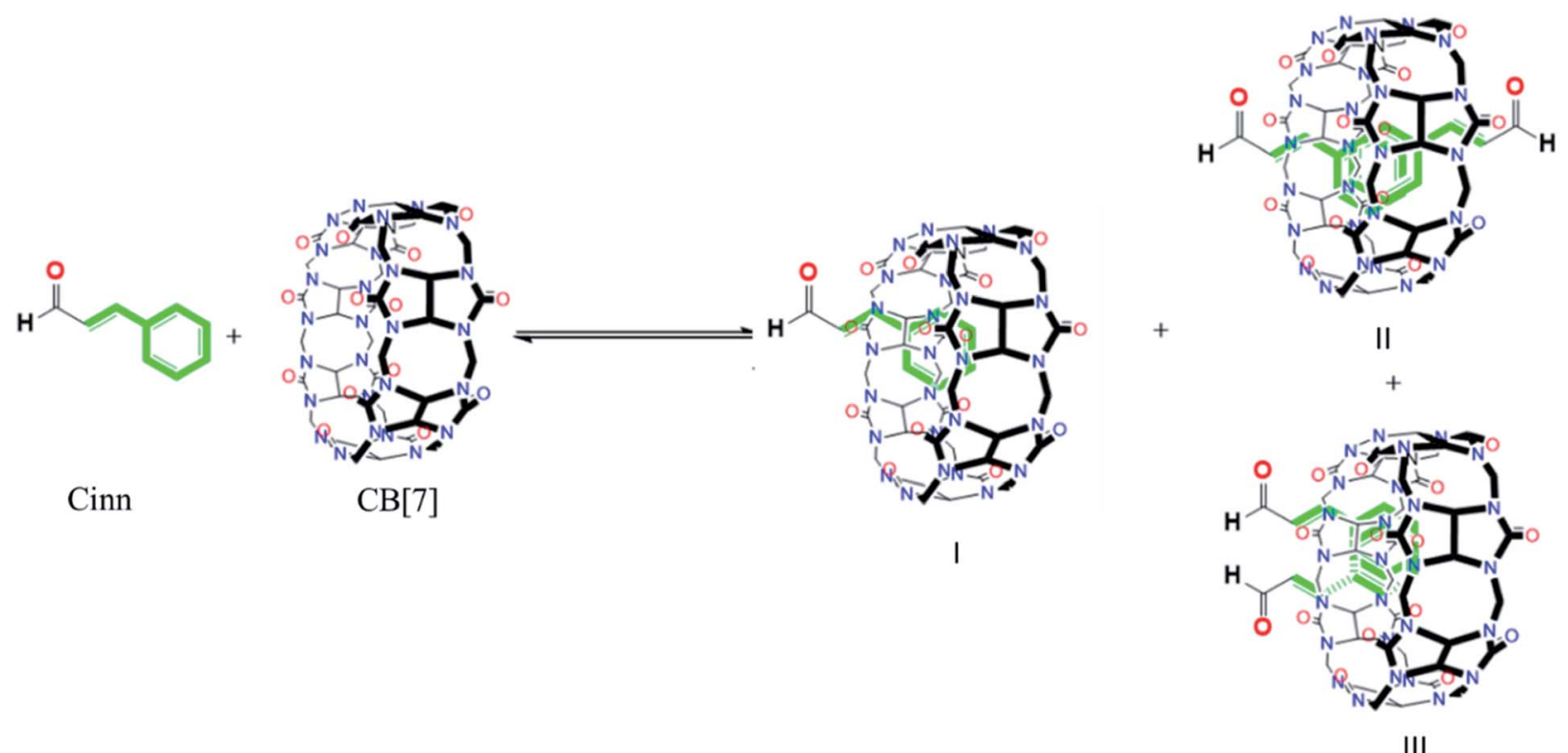

III

Fig. 7 The different possible complexes present at equilibrium. 


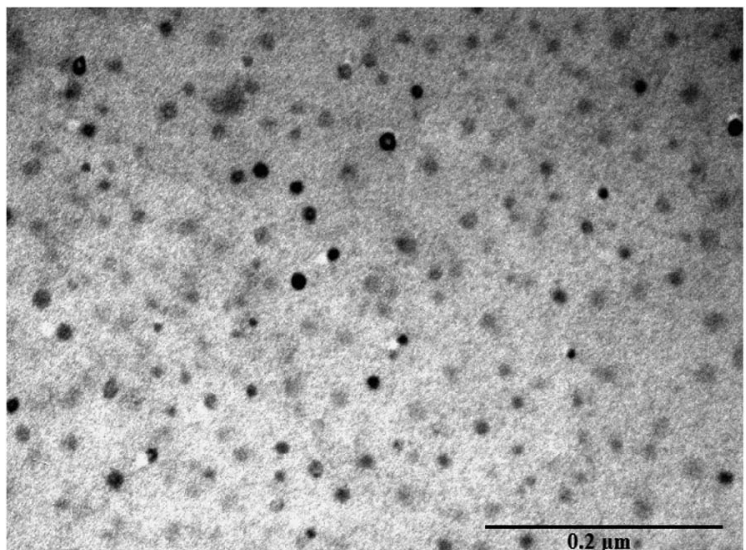

Fig. 9 Transmission electron microscope (TEM) images of CB[7]Cinn.

The charging the particles on the grid in TEM experiment to set and adhere to the sample separate the agglomerated particles into smaller one. ${ }^{42}$

\subsection{Computational study}

The ${ }^{1} \mathrm{H}$ NMR and optical measurements have demonstrated the formation of $1: 1$ and $1: 2$ inclusion complexes between $\mathrm{CB}[7]$ and Cinn. To confirm that, we relied on a density DFT level of theory using B3LYP functional with $6-31 \mathrm{G}(\mathrm{d}, \mathrm{p})$ basis set calculations to further assess the formation plausibility of both $1: 1$ and $1: 2 \mathrm{CB}[7]-C i n n$ host-guest complexes and propose a geometry of the inclusion complexes. Moreover, we investigated whether the inclusion complex occurs from the aldehyde or the phenyl side of Cinn (Fig. 10).

The analysis of the potential energy surfaces corresponding to aldehyde closing-up of the Cinn- $\mathrm{CB}[7]$ complex using the PBE0, M06-2X and B3LYP functional with 6-31G(d,p) basis set adapted similar energy behavior (Fig. 11). These energy diagrams showed unstable energy maxima at almost $3 \AA$ before the $7 \AA$ dissociation energy equilibrium distance. This result decreases the probability of formation of an inclusion complex from the aldehyde side of Cinn due to the repulsion interactions between $\mathrm{CB}[7]$ glycoluril carbonyl group with aldehyde side of Cinn which is in total agreement with ${ }^{1} \mathrm{H}$-NMR data that showed no alteration in shape or chemical shift of the aldehyde proton (Fig. 4a).

Clear stable energy minimums were observed at $3 \AA$ before the 7 distance $(\AA)$ dissociation energy equilibrium distance obtained from the different DFT functional caculations when considering the phenyl side orientation (Fig. 12). Similar energy curves were produced by the three DFT functional with a small variation in the calculated stabilization energy. These results indicate a strong attraction energy between the Cinn phenyl side and the hydrophobic cavity of $\mathrm{CB}[7]$ which is again in total agreement with ${ }^{1} \mathrm{H}$-NMR data (Fig. 4a).
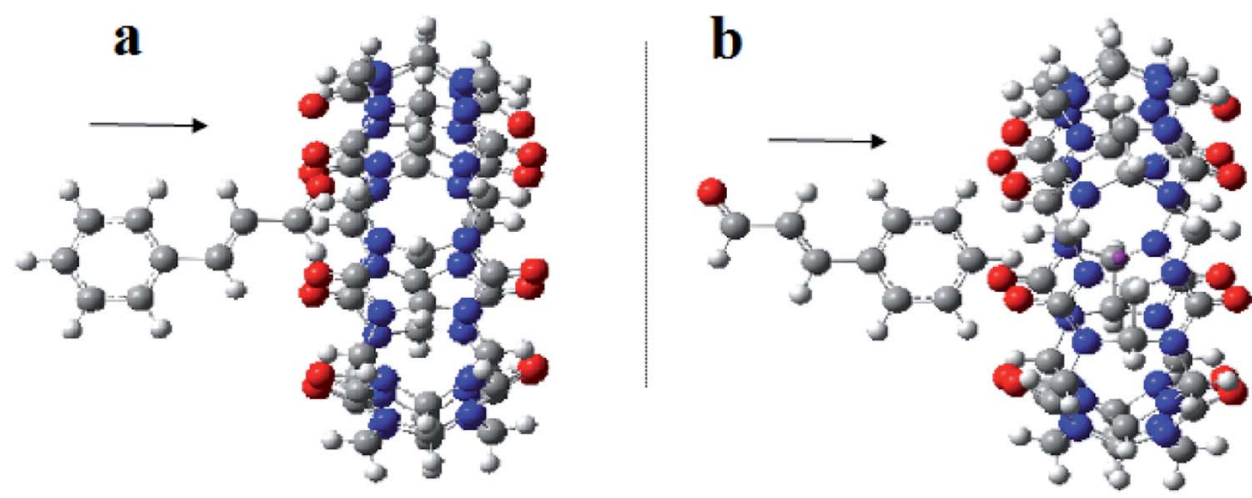

Fig. 10 A proposed structure for inclusion of Cinn into CB[7] with different orientations (a) aldehyde side and (b) phenyl side (by Gaussian view 09).

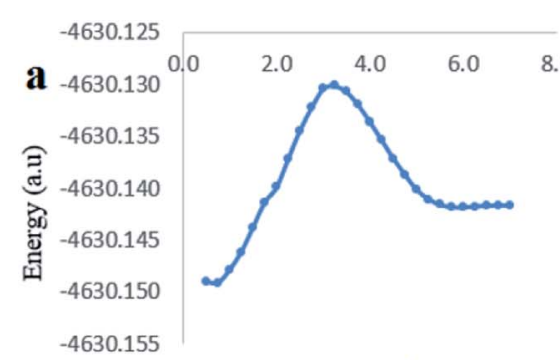

Distance $(\AA)$

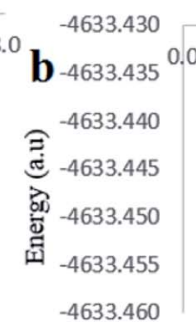

$-4633.460$

Fig. 11 Stability energy diagrams of the inclusion complexation of the Cinn from the aldehyde side into CB[7] at different DFT level of theory using (a) PBE0, (b) M06-2X and (c) B3LYP functional with 6-31G(d,p) basis set and CPMC model with solvation. 


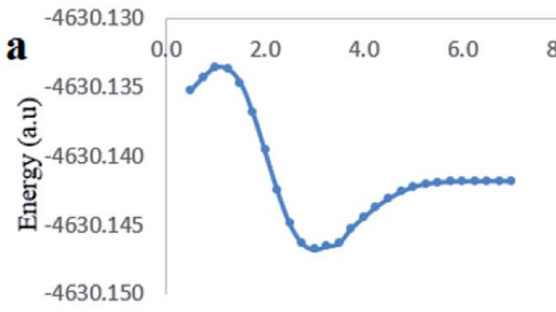

Distance $(\AA)$

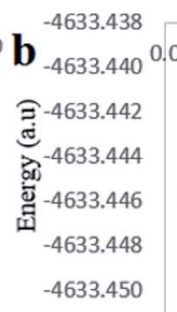

$-4633.452$

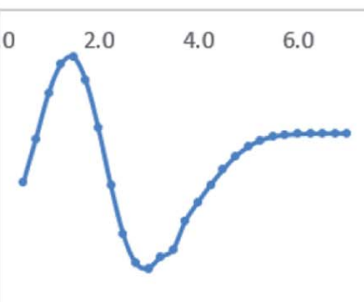

Distance $(\AA)$

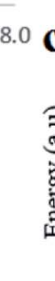

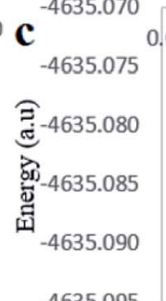

$-4635.095$

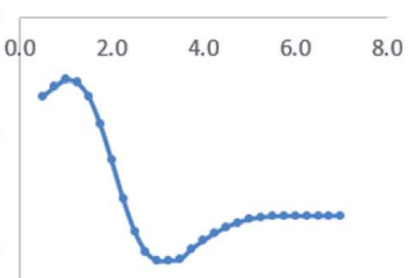

Distance $(\AA)$

Fig. 12 Stability energy diagrams of the inclusion complexation of the Cinn from the phenyl side into CB[7] at different DFT level of theory using (a) PBE0, (b) M06-2X and (c) B3LYP functional with 6-31G(d,p) basis set and CPMC model with solvation.

Table 1 A comparison between three DFT hybrid functionals theoretical calculations of different minimum energy, $r$ equilibrium and stabilization energy for inclusion of Cinn into CB[7] with different orientations (phenyl and aldehyde)

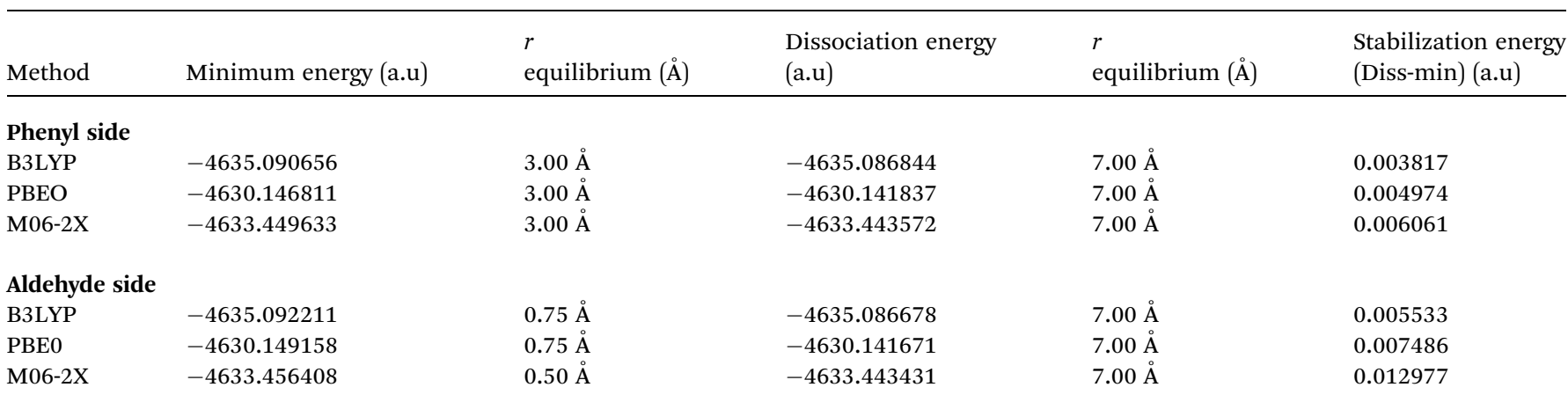

A comparison of the theoretical calculations of minimum energy, $r$ equilibrium and stabilization energy for inclusion of Cinn into $\mathrm{CB}[7]$ with different orientations, phenyl and aldehyde side, are summarize in Table 1 . The inclusion complex formation is more favorable from the Cinn phenyl side at $3 \AA$ due to the hydrophobic interaction, where it needs more close distance (0.5-0.75 $\AA$ ) of Cinn to form inclusion complex from the aldehyde side by the hydrogen bonding of the phenyl group with the glycoluril carbonyl of $\mathrm{CB}[7]$.

The highest stabilization energy was theoretically achieved using M06-2X functionals in both Cinn orientations. This indicates that DFT M06-2X functional is the most suitable for
Cinn and $\mathrm{CB}[7]$ interaction to build up the potential energy curve of the inclusion complex.

The last computational experiment was performed to investigate the theoretical ability of $\mathrm{CB}[7]$ to complex two molecules of Cinn from the phenyl side, a $1: 2$ stoichiometry, (Fig. 13a) using the DFT, B3LYP functional with 6-31G(d,p) basis set and CPMC/water solvent. The potential energy curve obtained from the $\mathrm{CB}[7]:$ Cinn, $1: 2$ stoichiometry, inclusion complex at the indicated level of theory and basis set showed more stable minima than observed for $1: 1$ stoichiometry (Fig. 13b). The stabilization energies for $1: 1$ and $1: 2$ stoichiometry were $10.02 \mathrm{~kJ} \mathrm{~mol}^{-1}$ and $11.32 \mathrm{~kJ} \mathrm{~mol}^{-1}$, respectively, at
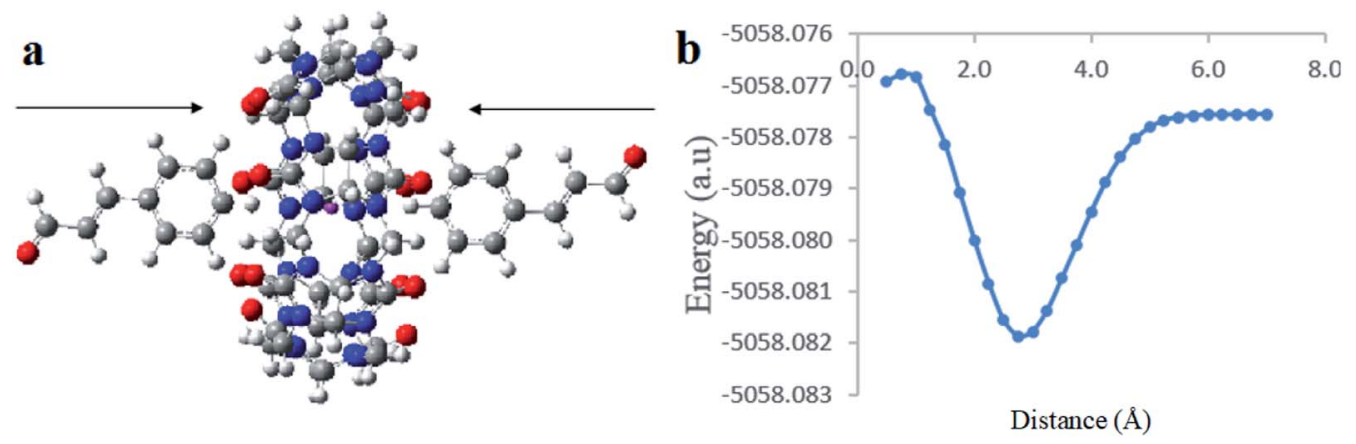

Fig. 13 (a) A proposed molecular structure for inclusion of two molecules of Cinn into CB[7] from the phenyl side (by Gaussian view 09). (b) Stability energy diagrams of the inclusion complexation of the two Cinn from the phenyl side into CB[7] at DFT level of theory using B3LYP functional with 6-31G(d,p) basis set and CPMC model with solvation. 

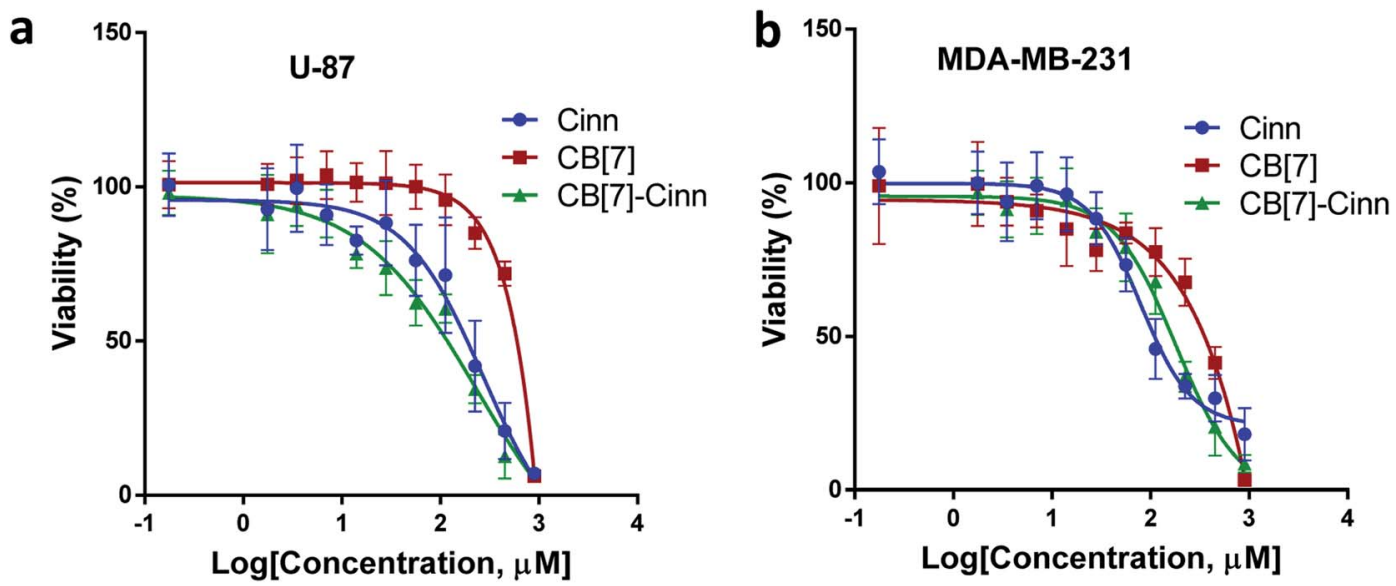

Fig. 14 The dose-response curve for (a) U-87 and (b) MDA-MB-231 cells treated with free Cinn, free CB[7] and CB[7]-Cinn. All cytotoxicity values represent the average $\pm \mathrm{SD}$ of three independent experiments.

$3.00 \AA$ distance, at the same level of theory and quantum parameters.

\subsection{In vitro cytotoxicity assay}

The cytotoxic activity of free Cinn, free $\mathrm{CB}[7]$ and $\mathrm{CB}[7]-\mathrm{Cinn}$ inclusion complex against U-87 and MDA-MB-231 cells were evaluated using cell viability assay (MTT). The IC $_{50}$ values were calculated after 72 hours of incubation with different concentrations of free Cinn, free $\mathrm{CB}[7]$ and $\mathrm{CB}[7]-\mathrm{Cinn}$ inclusion complex. The $\mathrm{IC}_{50}$ values for free Cinn, free $\mathrm{CB}[7]$ and $\mathrm{CB}[7]-$ Cinn inclusion complex were $240.17 \pm 32.46 \mu \mathrm{M}, 1000 \mu \mathrm{M}$ and $260.47 \pm 20.83 \mu \mathrm{M}$, respectively, against U-87 cells (Fig. 14a). The $\mathrm{IC}_{50}$ values for free Cinn, free $\mathrm{CB}[7]$ and $\mathrm{CB}[7]-\mathrm{Cinn}$ inclusion complex were $85.93 \pm 3.35 \mu \mathrm{M}, 1000 \mu \mathrm{M}$ and $176.3 \pm$ $7.79 \mu \mathrm{M}$, respectively, against MDA-MB-231 cells (Fig. 14b).

Our study confirmed that free $\mathrm{CB}[7]$ has no significant toxicity and $\mathrm{CB}[7]-\mathrm{Cinn}$ inclusion complex and the free Cinn showed antiproliferative effect against both cell lines. Almost no change in $\mathrm{IC}_{50}$ between free Cinn and $\mathrm{CB}[7]-\mathrm{Cinn}$ inclusion complex against U-87 cells, while the cytotoxicity was decreased by half with MDA-MB-231 cells. The slight variation in the cytotoxicity of $\mathrm{CB}[7]-\mathrm{Cinn}$ inclusion complex in the two cell lines related to varied characteristics of different cell lines and may be due to the probability of the two types of stoichiometry that may increase or decrease the concentration of the complexed Cinn. In addition, the $\mathrm{CB}[7]$ new complex with hydrophobic Cinn increases the aqueous solubility of the drug and/or drug permeability which could enhance Cinn bioactivity.

The anti-tumor effect of Cinn has been widely investigated against different cancers both in vitro and in vivo. ${ }^{8}$ Lee et al., 1999 reported the antiproliferative effect of Cinn against 29 cancer cell lines in vitro. ${ }^{43}$ Several reports described that Cinn exerts the anti-tumor effect through induction apoptosis and cell cycle arrest. ${ }^{44-47}$ Moreover, the combination of Cinn with other chemotherapies showed a higher anti-tumor effect compared to single drug use. ${ }^{47,48} \mathrm{~A}$ further study to investigate the combination of $\mathrm{CB}[7]$-Cinn inclusion complex with other chemotherapies in vitro and in vivo can be of great value.

\section{Conclusions}

The interactions between $\mathrm{CB}[7]$ and Cinn, to increased Cinn stability, have been studied in detail by ${ }^{1} \mathrm{H}-\mathrm{NMR}$ spectroscopy, UV-vis absorption spectroscopy and DFT quantum calculation. The ${ }^{1} \mathrm{H}$-NMR spectra and the spectrophotometric studies proved the $1: 1$ and $1: 2$ stoichiometries. Moreover, the DFT calculation analysis indicated that $\mathrm{CB}[7]$ selectively interacted with Cinn from the phenyl moiety.

Our study suggests that $\mathrm{CB}[7]$-Cinn inclusion complex can be an effective as free Cinn against MDA-MB-231 and U-87 cancer cell lines that provide $\mathrm{IC}_{50}$ values $176.3 \pm 7.79 \mu \mathrm{M}$, $260.47 \pm 20.83 \mu \mathrm{M}$, respectively. This study suggests that $\mathrm{CB}[7]-$ based pharmaceutical formulation is a promising drug delivery system that may improve the stability and bioavailability of hydrophobic drugs in vitro and in vivo. Moreover, the investigation of the synergistic effect between Cinn and other chemotherapies can provide a valuable combination therapy through decreasing the required doses of the cytotoxic drugs and may reduce the unwanted side-effects.

\section{Conflicts of interest}

There are no conflicts to declare.

\section{Acknowledgements}

The authors would like to thank the Deanship of Academic Research (DAR) at The University of Jordan (UJ) for their financial support.

\section{References}

1 Z. Liu, J. T. Robinson, X. Sun and H. Dai, J. Am. Chem. Soc., 2008, 130, 10876-10877.

2 M. Ferrari, Nat. Rev. Cancer, 2005, 5, 161-171.

3 P. Domadia, S. Swarup, A. Bhunia, J. Sivaraman and D. Dasgupta, Biochem. Pharmacol., 2007, 74, 831-840. 
4 Y.-T. Tung, M.-T. Chua, S.-Y. Wang and S.-T. Chang, Bioresour. Technol., 2008, 99, 3908-3913.

5 S.-J. Wu and L.-T. Ng, Food Chem. Toxicol., 2007, 45, 24462453.

6 S. Jiang, J.-N. Li and Z.-T. Jiang, Eur. Food Res. Technol., 2010, 230, 543-550.

7 A. Dutta and A. Chakraborty, J. Toxicol., 2018, 2018, 8978731.

8 S. H. Hong, I. A. Ismail, S. M. Kang, D. C. Han and B. M. Kwon, Phytother. Res., 2016, 30, 754-767.

9 R. V. Olsen, H. H. Andersen, H. G. Moller, P. W. Eskelund and L. Arendt-Nielsen, Eur. J. Pain, 2014, 18, 1333-1342.

10 Y. Huang, S.-F. Xue, Q.-J. Zhu and T. Zhu, Supramol. Chem., 2008, 20, 279-287.

11 W. Alshaer, M. Zraikat, A. Amer, H. Nsairat, Z. Lafi, D. A. Alqudah, E. Al Qadi, T. Alsheleh, F. Odeh, A. Alkaraki, M. Zihlif, Y. Bustanji, E. Fattal and A. Awidi, RSC Adv., 2019, 9, 30976-30988.

12 J.-H. Li, N. Zhang, X.-T. Li and J.-Y. Wang, J. Inclusion Phenom. Mol. Recognit. Chem., 1997, 28, 95-103.

13 M. E. Carlotti, S. Sapino, R. Cavalli, M. Trotta, F. Trotta and K. Martina, J. Inclusion Phenom. Macrocyclic Chem., 2007, 57, 445-450.

14 J. He and W. Li, J. Inclusion Phenom. Macrocyclic Chem., 2009, 63, 61-68.

15 L. E. Hill, C. Gomes and T. M. Taylor, LWT-Food Sci. Technol., 2013, 51, 86-93.

16 F. Zarei baygi, N. Farhadian, B. Malaekeh-Nikouei and Z. Maghsoud, RSC Adv., 2016, 6, 111517-111525.

17 M. Y. Hur, I. Hwang and K. Kim, in Cucurbiturils and Related Macrocycles, The Royal Society of Chemistry, 2020, pp. 1-14, DOI: 10.1039/9781788015967-00001.

18 A. I. Day, R. J. Blanch, A. P. Arnold, S. Lorenzo, G. R. Lewis and I. Dance, Angew. Chem., Int. Ed. Engl., 2002, 41, 275-277.

19 J. Lagona, P. Mukhopadhyay, S. Chakrabarti and L. Isaacs, Angew. Chem., Int. Ed. Engl., 2005, 44, 4844-4870.

20 V. D. Uzunova, C. Cullinane, K. Brix, W. M. Nau and A. I. Day, Org. Biomol. Chem., 2010, 8, 2037-2042.

21 S. Karcher, A. Kornmüller and M. Jekel, Water Res., 2001, 35, 3309-3316.

22 A. Day, A. P. Arnold, R. J. Blanch and B. Snushall, J. Org. Chem., 2001, 66, 8094-8100.

23 A. L. Koner and W. M. Nau, Supramol. Chem., 2007, 19, 5566.

24 J. S. Negi and S. Singh, Carbohydr. Polym., 2013, 92, 18351843.

25 C. Tablet, I. Matei and M. Hillebrand, The Determination of the Stoichiometry of Cyclodextrin Inclusion Complexes by Spectral Methods: Possibilities and Limitations, INTECH Open Access Publisher, 2012.

26 K. Srinivasan, T. Stalin and K. Sivakumar, Spectrochim. Acta, Part A, 2012, 94, 89-100.

27 V. A. Marcolino, G. M. Zanin, L. R. Durrant, T. Benassi Mde and G. Matioli, J. Agric. Food Chem., 2011, 59, 3348-3357.
28 F. Odeh, H. Nsairat, W. Alshaer, S. Alsotari, R. Buqaien, S. Ismail, A. Awidi and A. Al Bawab, RSC Adv., 2019, 9, 37148-37161.

29 C. Nicolazzi, S. Abdou, J. Collomb, A. Marsura and C. Finance, Bioorg. Med. Chem., 2001, 9, 275-282.

30 R. Rajaram, K. N. Sundararajalu and S. Meenakshisundaram, J. Fluoresc., 2011, 21, 521-529.

31 S. G. M. Ong, L. C. Ming, K. S. Lee and K. H. Yuen, Pharmaceutics, 2016, 8, 25.

32 M. Frisch, G. Trucks, H. Schlegel, G. Scuseria, M. Robb, J. Cheeseman, G. Scalmani, V. Barone, B. Mennucci, G. Petersson, H. Nakatsuji, M. Caricato, X. Li, H. Hratchian, A. Izmaylov, J. Bloino, G. Zheng, J. Sonnenberg, M. Hada and D. Fox, Gaussian Inc.Wallingford CT, 2009.

33 T. Mosmann, J. Immunol. Methods, 1983, 65, 55-63.

34 N. Horiuchi, K. Nakagawa, Y. Sasaki, K. Minato, Y. Fujiwara, K. Nezu, Y. Ohe and N. Saijo, Cancer Chemother. Pharmacol., 1988, 22, 246-250.

35 Z. Hadian, M. Maleki, K. Abdi, F. Atyabi, A. Mohammadi and R. Khaksar, Iran. J. Pharm. Res., 2018, 17, 39-51.

36 V. Sindelar, S. E. Parker and A. E. Kaifer, New J. Chem., 2007, 31, 725-728.

37 A. B. Hegge, M. Masson, S. Kristensen and H. H. Tonnesen, Pharmazie, 2009, 64, 382-389.

38 A. L. Koner, I. Ghosh, N. i. Saleh and W. M. Nau, Can. J. Chem., 2011, 89, 139-147.

39 E. Olson and P. Buhlmann, J. Org. Chem., 2011, 76, 84068412.

40 J. S. Renny, L. L. Tomasevich, E. H. Tallmadge and D. B. Collum, Angew. Chem., Int. Ed. Engl., 2013, 52, 1199812013.

41 D. L. Romano, J. E. Marcy and R. C. Williams, Master thesis, Virginia Polytech Institute and State University, 2008.

42 M.-J. Choi, U. Ruktanonchai, A. Soottitantawat and S.-G. Min, Food Res. Int., 2009, 42, 989-997.

43 C. W. Lee, D. H. Hong, S. B. Han, S. H. Park, H. K. Kim, B. M. Kwon and H. M. Kim, Planta Med., 1999, 65, 263-266.

44 S. K. Rad, M. S. Kanthimathi, S. N. Abd Malek, G. S. Lee, C. Y. Looi and W. F. Wong, PLoS One, 2015, 10, e0145216.

45 P. Kubatka, M. Kello, K. Kajo, M. Samec, K. Jasek, D. Vybohova, S. Uramova, A. Liskova, V. Sadlonova, L. Koklesova, R. Murin, M. Adamkov, K. Smejkal, E. Svajdlenka, P. Solar, S. M. Samuel, M. Kassayova, T. K. Kwon, P. Zubor, M. Pec, J. Danko, D. Busselberg and J. Mojzis, Molecules, 2020, 25(6), 1399.

46 J. Li, Y. Teng, S. Liu, Z. Wang, Y. Chen, Y. Zhang, S. Xi, S. Xu, R. Wang and X. Zou, Oncol. Rep., 2016, 35, 1501-1510.

47 A. Abbasi, M. Hajialyani, L. Hosseinzadeh, F. Jalilian, P. Yaghmaei, S. Jamshidi Navid and H. Motamed, Results Pharma Sci., 2020, 15, 26-35.

48 S. Y. Shin, H. Jung, S. Ahn, D. Hwang, H. Yoon, J. Hyun, Y. Yong, H. J. Cho, D. Koh, Y. H. Lee and Y. Lim, Bioorg. Med. Chem., 2014, 22, 1809-1820. 